\title{
Clinical Adverse Events after Endoscopic Resection for Colorectal Lesions: A Meta-Analysis on the Antibiotic Prophylaxis
}

\author{
Davide La Regina ${ }^{a}$ Francesco Mongellia Alberto Fasolib Gianluca Lollo ${ }^{b}$ \\ Marcello Ceppic ${ }^{c}$ Andrea Saporito ${ }^{d}$ Fabio Garofalo ${ }^{e}$ Matteo Di Giuseppe ${ }^{a}$ \\ Antonjacopo Ferrario di Tor Vajana ${ }^{a}$ \\ ${ }^{a}$ Department of Surgery, Ospedale Regionale di Bellinzona e Valli, Bellinzona, Switzerland; b Department of \\ Gastroenterology, Ospedale Regionale di Bellinzona e Valli, Bellinzona, Switzerland; ' Unit of Clinical Epidemiology,

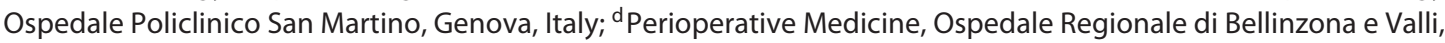 \\ Bellinzona, Switzerland; ${ }^{e}$ Department of Surgery, Ospedale Regionale di Lugano, Lugano, Switzerland
}

\author{
Keywords \\ Endoscopic mucosal resection - Endoscopic submucosal \\ dissection · Antibiotic prophylaxis · Postoperative \\ complications
}

\begin{abstract}
Background: Post-polypectomy coagulation syndrome (PECS) is a well-known adverse event after endoscopic polypectomy for colorectal lesions. To date, there are no standardized guidelines for the antimicrobial prophylaxis. The aim of this meta-analysis is to evaluate the usefulness of antibiotics in patients undergoing endoscopic mucosal or submucosal resections. Methods: A comprehensive literature search of PubMed, MEDLINE, EMBASE, and Web of Science databases was carried out according to the Preferred Reporting Items for Systematic Reviews and Meta-Analyses guidelines. Studies investigating the role of prophylactic antibiotic administration in reducing the PECS after endoscopic polypectomy were considered. The terms used to search were ("antimicrobial"OR"antibiotics"OR"prophylaxis"OR"pr ophylactic") AND ("resection"OR"polypectomy"OR"dissecti on") AND ("endoscopic"OR"mucosal"OR"submucosal") AND ("colon"OR"colorectal"OR"colonic"OR"rectum"). Data of in-
\end{abstract}

cluded studies were collected and analysed. Results: The literature search revealed 262 articles, 3 of whom were randomized trials and one was a retrospective study. Patients included were 850 (548 treated with antibiotics and 302 received no treatment). The overall incidence rate was 2.4 and $19.9 \%$ in treatment and control groups, respectively. The pooled analysis showed a reduction of $83 \%$ of postoperative events in the antibiotics group (relative risk $0.181 ; 95 \% \mathrm{Cl}$ $0.100-0.326, p<0.001)$. Conclusions: In our meta-analysis, the antibiotic prophylaxis showed a positive effect in reducing the incidence of postoperative adverse events other than perforation and bleeding in patients treated with endoscopic mucosal resection and endoscopic submucosal dissection for colorectal lesions. Despite the low-level of evidence of this meta-analysis, the antibiotic prophylaxis should be taken into account. Further multicenter, large-sample, randomized controlled studies are needed to confirm our results and to evaluate whether specific subgroups of patients could actually benefit from an antibiotic prophylaxis.

(c) 2019 S. Karger AG, Basel

D. La Regina and F. Mongelli contributed equally to the paper.

\section{KARGER}

(C) 2019 S. Karger AG, Basel

E-Mail karger@karger.com

www.karger.com/ddi
Francesco Mongelli, MD

Department of Surgery

Ospedale Regionale di Bellinzona e Valli

via Ospedale, $\mathrm{CH}-6500$ Bellinzona (Switzerland)

E-Mail francesco.mongelli@ mail.com 


\section{Introduction}

Perforation and bleeding are recognized complications of the endoscopic resection of colorectal polyps [1]. However, several other adverse events can occur. Post procedural abdominal pain and fever can lead to hospitalization and in-patient treatment for several days [2]. Although not uniformly described, post-polypectomy coagulation syndrome (PECS) is a well-known adverse event occurring in around $1 \%$ of cases after endoscopic mucosal resection (EMR), and in 7-8\% of cases after endoscopic submucosal dissection (ESD) [3]. Thought to be due to electrical current and thermal injuring extending into the muscularis propria and serosa, PECS results in a transmural burn at the site of polypectomy. Moreover, the exposed surface after the polypectomy and the inflammatory reaction may also contribute to its development. PECS occurs more often in females, ESD operation time $>90 \mathrm{~min}$, lesions of the cecum, and ascending colon,

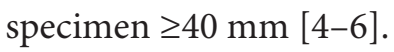

At present, there are no standardized guidelines for the antimicrobial prophylaxis for the endoscopic polypectomy for colorectal lesions, so that, the antibiotic use commonly depends on the endoscopist's preference and experience [7]. Antibiotic therapy may be useful to reduce the superinfection and the inflammatory response within the area of resected polyps. To date, the usefulness of antibiotics in preventing adverse events other than perforation and bleeding after endoscopic resection of colorectal polyps was not adequately investigated. The purpose of the study was to compare the incidence of PECS in patients undergoing EMR and ESD for colorectal polyps, treated with or without perioperative antibiotic therapy. To clarify the matter, we conducted a review of the available literature.

\section{Methods}

The meta-analysis was based on previous published studies. No ethical approval and patient consent were required.

Search Strategy and Selection Criteria

A comprehensive computer literature search of PubMed, MEDLINE, EMBASE, and Web of Science databases was carried out by 2 researchers independently, in order to find relevant published articles (last search was updated on June 15, 2019) according to the Preferred Reporting Items for Systematic Reviews and MetaAnalyses guidelines [8]. The terms used to search were:

("antimicrobial"OR"antibiotics"OR"prophylaxis"OR"prophyl actic") AND ("resection"OR"polypectomy"OR"dissection") AND ("endoscopic"OR"mucosal"OR"submucosal") AND ("colon"OR"
colorectal"OR"colonic"OR"rectum"). Finally, we searched for additional eligible trials in reference lists of retrieved publications and relevant meta-analyses. No language restrictions were set.

\section{Data Collection and Quality Assessment}

Studies or subsets in studies investigating the role of the prophylactic antibiotic administration after endoscopic polypectomies were considered. Case reports, small case series, review articles, letters, editorials, and conference proceedings were excluded. The following inclusion criteria were applied to select studies for this meta-analysis: patients treated for endoscopic polypectomy for benign or malignant lesions, randomized controlled trials investigating the post-procedural antibiotic administration, adequate data regarding the post-procedural infection rate. Exclusion criteria were coagulation deficiency, pregnancy, concurrent antibiotic therapy, studies published with insufficient information.

We extracted study characteristics (author name, publication year, country, sample size, age, study design, inclusion and exclusion criteria, antibiotics, method of randomization, blinding), primary outcomes (post-procedural infection rate), and risk of bias. Any disagreement between reviewers was resolved by discussion. Only studies providing such complete information were finally included in the meta-analysis.

The quality assessments of studies included in the meta-analysis were independently performed by 2 reviewers according to the Study Quality Assessment of Controlled Intervention Studies NHLBI [9]. Ultimately, the overall quality of evidence was graded with Grading of Recommendations, Assessment, Development and Evaluations (GRADE approach) [10]. The quality of evidence was rated with a scale of $4-1$ ( $4=$ high, $3=$ moderate, $2=$ low, and $1=$ very low). Five factors could reduce by 1 or 2 the initial quality of evidence (risk of bias, inconsistency, indirectness, imprecision and publication bias) of selected studies.

\section{Statistical Methods}

Study-specific relative risk's (RR) along with 95\% CI were estimated from the number of patients and the number of events in each arm as reported from each study. Pooled estimate of RR's was obtained by applying the random effects model following DerSimonian et al. [11]. A forest plot was generated to display results. To obtain a quantitative measure of the degree of inconsistency in the results of studies, we calculated a Higgins' $I^{2}$ index [12]. Publication bias was assessed by visual inspection of funnel plots and with Harbord's asymmetry test [13]. To assess whether the pooled estimate was strongly affected by one of the studies included in the meta-analysis, the sensitivity analysis was performed. This analysis iteratively recalculated the pooled RR estimate after exclusion of each study at a time. STATA software was used for all statistical analyses and for the generation of forest plot (Stata Statistical Software: Release 14.2; StataCorp LP: College Station, TX, USA, 2015).

\section{Results}

\section{Literature Search}

The comprehensive computer literature search from PubMed, MEDLINE, EMBASE, and Web of Science databases revealed 262 articles. Gray literature was searched
La Regina et al. 
Fig. 1. Flow chart of the literature search according to Preferred Reporting Items for Systematic Reviews and Meta-Analyses Guidelines.

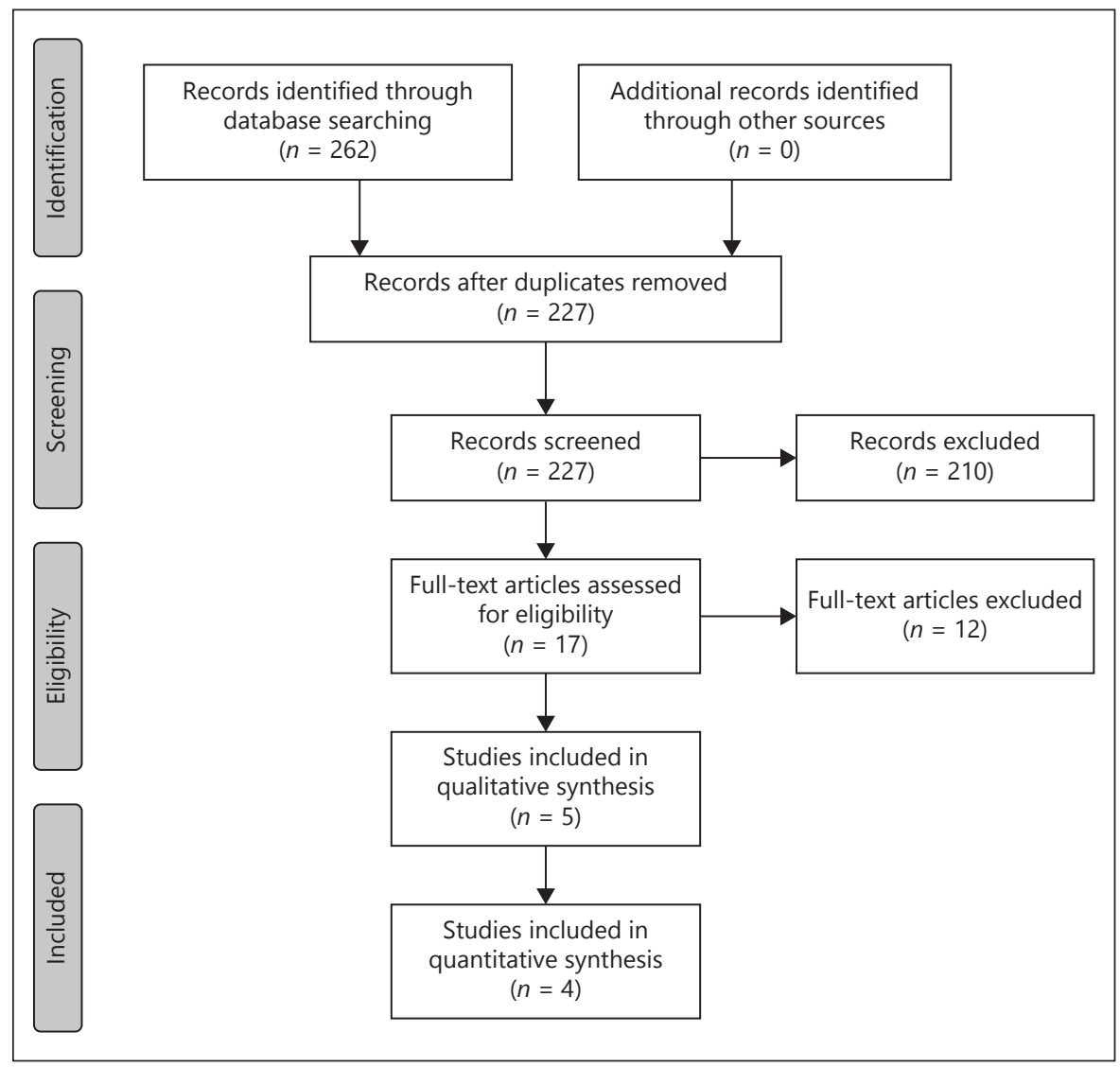

on Google Scholar and OpenGrey and no relevant studies investigating the role of the antibiotics in patients undergoing ESD or EMR for colorectal polyps were identified. Results were matched 35 duplicates were removed. Reviewing titles and abstracts, 210 records were excluded did not match the main topic. Seventeen articles were assessed for eligibility and 12 were excluded as they were reviews, editorials, letters or case reports. Five studies were considered in the qualitative synthesis [14-18]; finally, 3 randomized trial and a retrospective study were selected and considered eligible for the quantitative analysis [14-17]. No additional studies were found screening the references (Fig. 1).

\section{Study Characteristics}

Eligible studies were 3 randomized trials and a retrospective study published from 2015 to 2017 . Among randomized trials, one study was placebo controlled [14] and 2 studies $[15,16]$ were untreated controlled. The study of Lee et al. [14] considered ESD only for polyps with a mean size of $20.56 \pm 8.9 \mathrm{~mm}$ (antibiotics group) and $20.42 \pm$ $8.22 \mathrm{~mm}$ (control group). The polyps morphology was: laterally spreading nongranular $26 \%$ (flat elevated $20 \%$, pseudo-depressed 6\%), laterally spreading granular $45 \%$ (homogeneous 23\%, nodular mixed 22\%) and polypoid 29\%. Zhang et al. [15] considered EMR and ESD for polyps with a size $<10 \mathrm{~mm}$ in 20.5 and $17.8 \%$ of cases, $10-20 \mathrm{~mm}$ in 56.1 and $60.7 \%$ of cases and $>20 \mathrm{~mm}$ in 23.4 and $21.5 \%$ of cases in the antibiotic and control group respectively. In this study, polyps were pedunculated in $14 \%$ of cases, subpeduncolated in $29 \%$, sessile in $21.5 \%$, flat elevated in $27.1 \%$ and flat in $8.5 \%$ of cases. Shi et al. [16] considered benign protruded colon polyps $<20 \mathrm{~mm}$ treated with EMR, flat and laterally spreading polyps were excluded. Muro et al. [17] considered patients undergoing ESD for colorectal lesions but no further information on the size and the morphology were reported.

The antibiotics regimens and duration of postoperative treatments were evaluated in the selected trials. In the treatment arm, the antibiotic administered by Lee et al. [14] was ampicillin and/or sulbactam $1 \mathrm{~h}$ preoperatively and after 7 and $15 \mathrm{~h}$ postoperatively. Zhang et al. [15] administered cefuroxime $30 \mathrm{~min}$ before and $6 \mathrm{~h}$ after the procedure. In the trial of Shi et al. [16], the patients were treated with levofloxacin or ceftazidime injection for 3 days postoperatively. In the study of Muro et al. [17], the 
Table 1. Characteristics and results of included studies

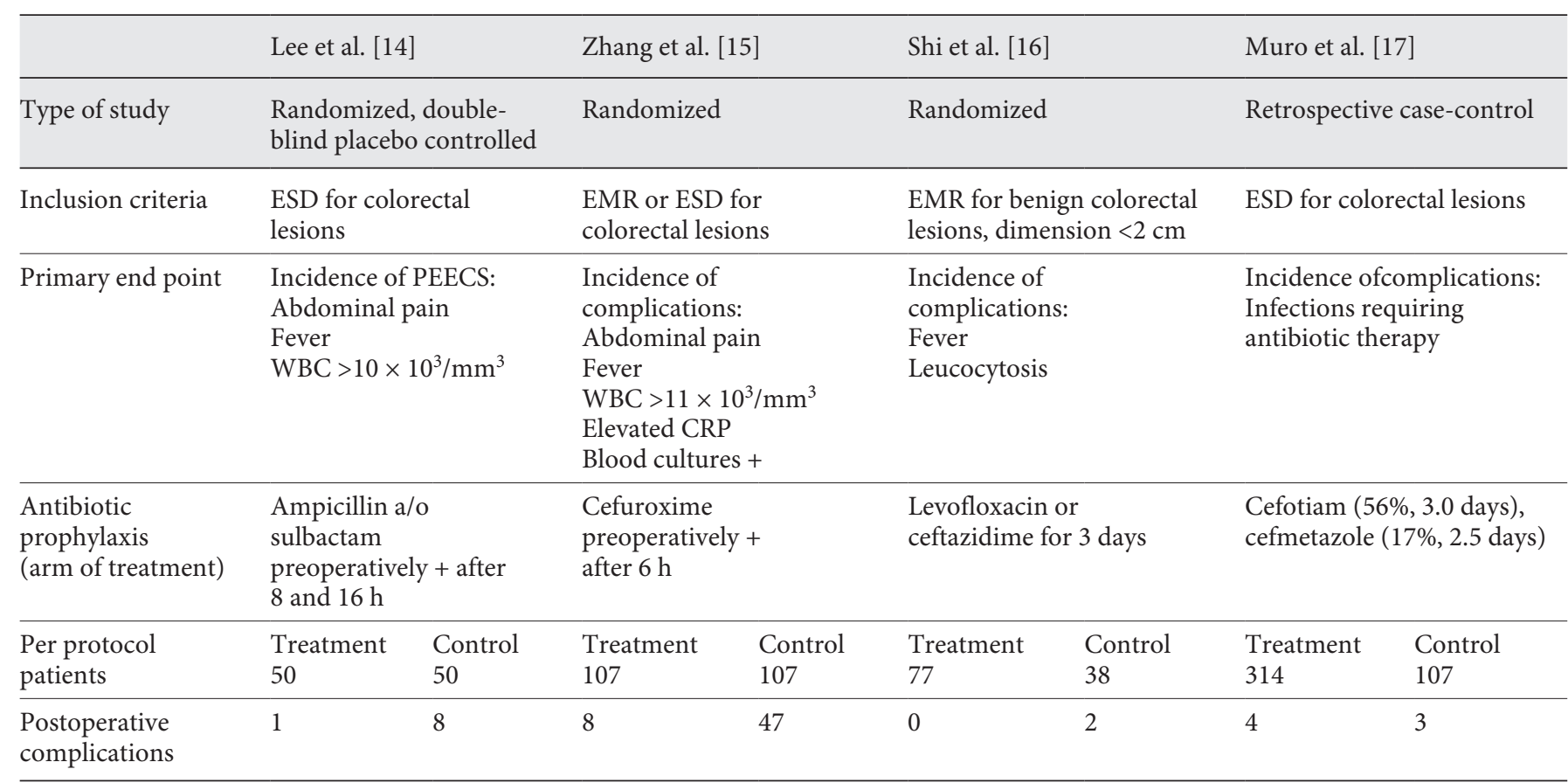

ESD, endoscopic submucosal dissection; EMR, endoscopic mucosal resection; PEECS, post-ESD electrocoagulation syndrome; WBC, white blood cells; CRP, C-reactive protein.

patients received different antibiotic regimens, with cefotiam being the most commonly prescribed antimicrobial prophylaxis (56\%, median dosing period of 3.0 days) and cefmetazole the second one (17\%, median dosing period of 2.5 days). Among the selected studies, the primary endpoint was the evaluation of the incidence of postoperative complications other than perforations and bleeding. In particular, Lee et al. [14] defined the post-ESD electrocoagulation syndrome as abdominal pain or fever or white blood cells $>10 \times 10^{3} / \mathrm{mm}^{3}$, while Zhang et al. [15] considered the presence of abdominal pain, fever, leucocytosis, elevated C-reactive protein, positive blood cultures, Shi et al. [16] considered fever and leucocytosis as postoperative complications, and Muro et al. [17] defined postoperative infections as patients who were treated with antimicrobial therapy for postoperatively. The characteristics of the included studies are presented in Table 1.

The risk of bias among the included studies is reported in Figure 2. According to the Study Quality Assessment of Controlled Intervention Studies NHLBI [9], the study of Lee et al. [14] and Zhang et al. [15] was categorized "good," the study of Shi et al. [16] was categorized "fair" and the study of Muro et al. [17] was categorized "poor". According to the GRADE approach, due to limitations in studies quality (lack of blinding rated down by 1 ) and sparse data (lack of directness rated down by 1), in this meta-analysis the overall quality of evidence was judged "low".

\section{Statistical Results}

In our meta-analysis, 850 patients were included, of whom 548 were treated with antibiotics and 302 received no treatment. All studies showed a reduction in the rate of postoperative adverse events. The overall incidence rate was 2.4 and $19.9 \%$ in treatment and control groups, respectively. Two studies reported a statistically significant difference among groups, so that heterogeneity is null. The pooled analysis showed a reduction of $83 \%$ (RR $0.181 ; 95 \%$ CI $0.100-0.326, p<0.001)$. The statistical results with Forest plot are reported in Figure 3. The funnel plot (Fig. 4) did not show evident asymmetries, and the Harbord test was not significant $(p=0.274)$.

\section{Discussion}

Colonoscopy is a very effective screening method for colorectal cancer. The endoscopic removal of precancerous polyps and the early detection of the disease 
Fig. 2. Risk of bias assessment of included studies according to Study Quality Assessment of Controlled Intervention Studies NHLBI.

Fig. 3. Forest plot of the statistical analysis with relative risk (RR), 95\% CI and the subtotal $I^{2}$ for the included studies.

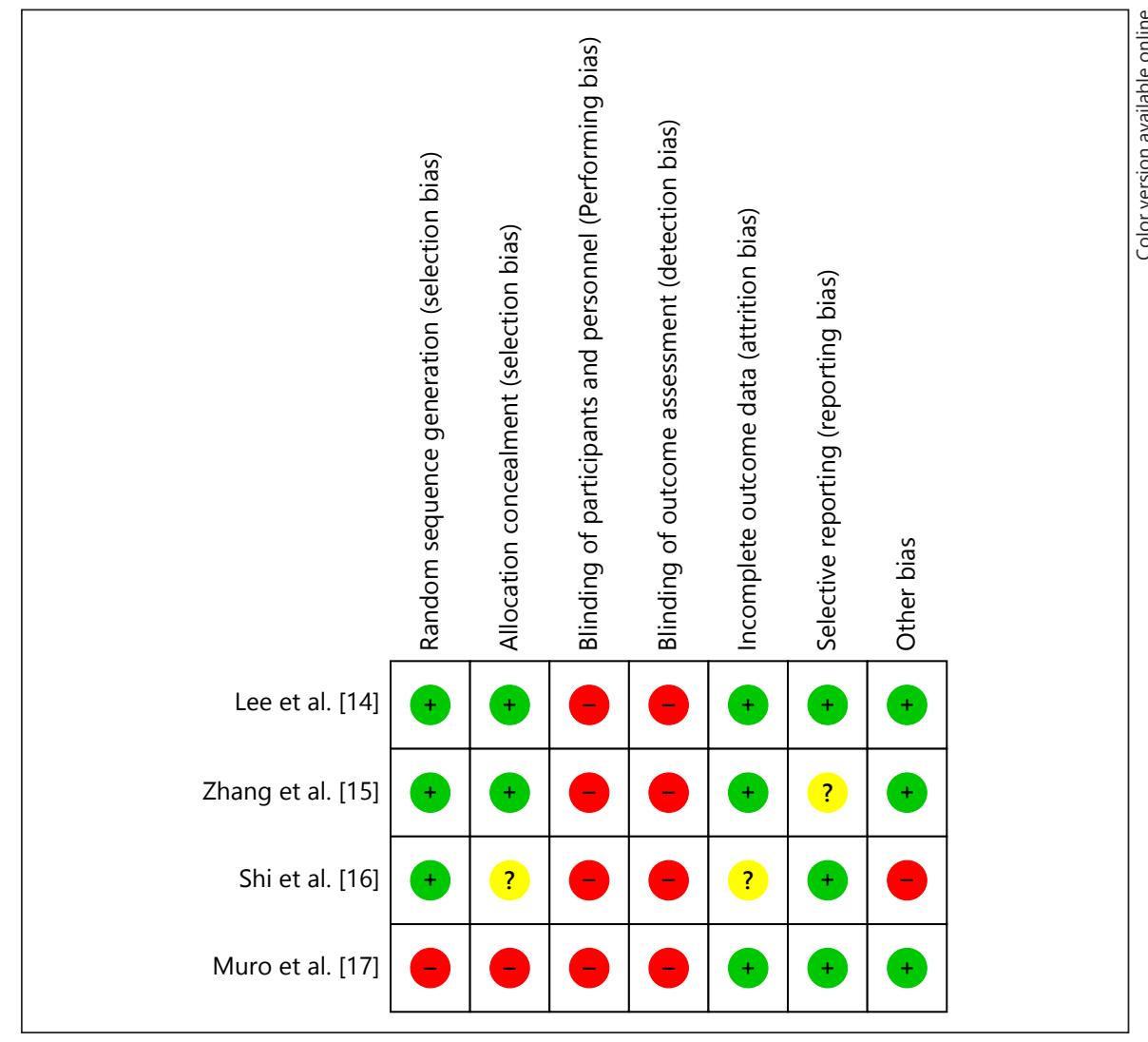

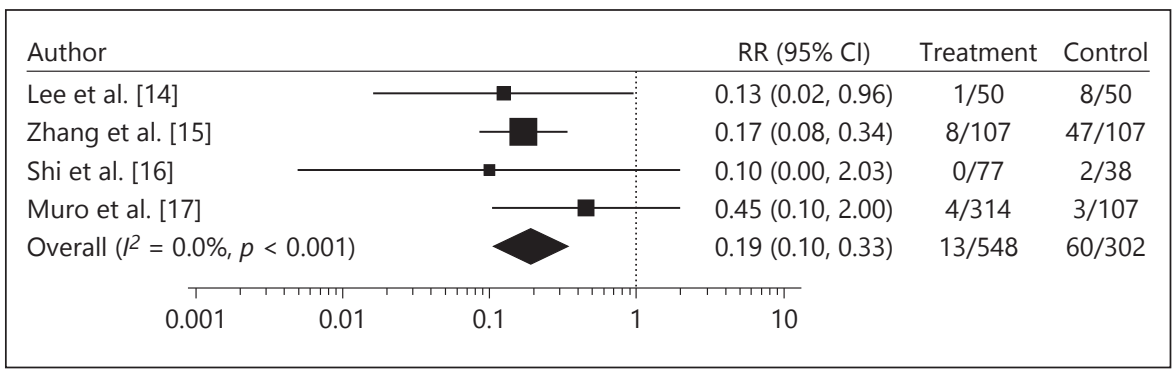

at a localized stage strongly reduce the risk of colorectal cancer [19]. In a recent systematic review, postcolonoscopy complications were defined as perforations, bleeding, or mortality occurring within 30 day [1]. However, further adverse events, other than perforation and bleeding, may occur after colonoscopy. In the clinical practice, abdominal pain and fever, eventually associated with leucocytosis, can occur after the endoscopic resection of colorectal polyps and be regarded as complications [2]. Snare polypectomy, EMR, and endoscopic submucosal resection are the 3 main techniques of removal [19]. The choice among these different techniques depends on the size, location, and shape of the polyp and on the expertise of the operator [19].

The use of an electrosurgical current increases the risk of complications [19]. A PECS was mentioned firstly in 1981 by Waye [20], who described symptoms resembling diverticulitis, appendicitis, or colonic perforation $6 \mathrm{~h}$ after polypectomy with electrocoagulation. Although not uniformly reported in the medical literature, PECS is commonly defined as the development of abdominal pain, fever, high white blood cell count, and peritoneal inflammation in the absence of bowel perforation after polypectomy with electrocoagulation [2]. In this respect, PECS has also been described as transmural burn syn- 


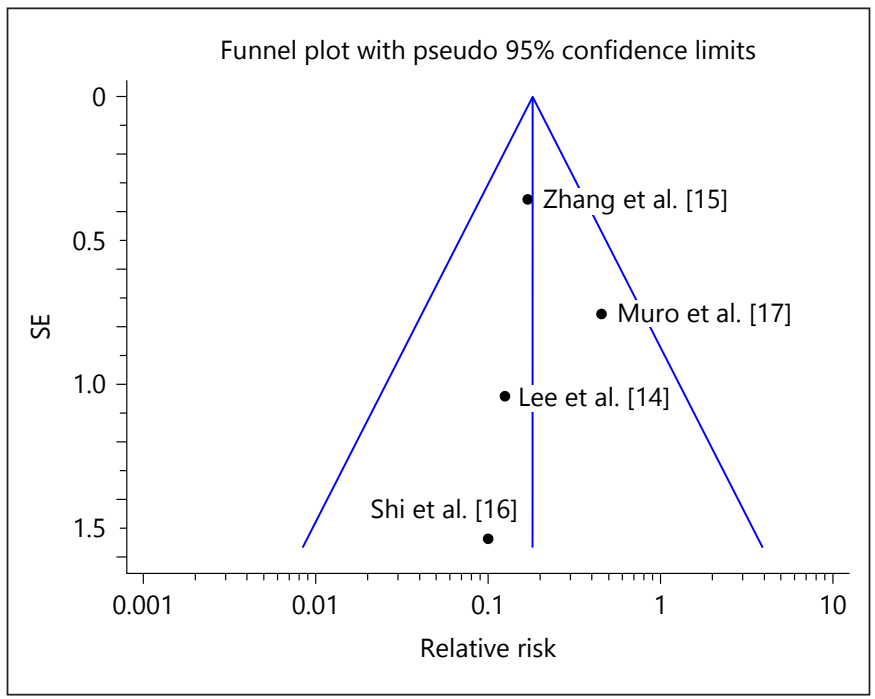

Fig. 4. Funnel-plot did not show asymmetries among included studies. $\mathrm{RR}$, relative risk.

drome. Supposedly, the electrocoagulation injury of the mucosal and submucosal layers causes a transmural burn with inflammation of the peritoneum, so that, the patient develops symptoms and signs of bowel perforation without its evidence on imaging studies [4]. PECS occurs in around $1 \%$ of cases after EMR and in 7-8\% of cases after ESD [3], more often in females, ESD operation time $>90 \mathrm{~min}$, lesions of the cecum and ascending colon, specimen $\geq 40 \mathrm{~mm}$. Unlike other post-colonoscopy complications such as perforation and bleeding, PECS can be usually treated conservatively with fluids, pain killers, and antibiotics [2]. Although hospitalization is not always necessary, the median duration of the in-patient treatment amounts to 5.5 days [2]. Besides being a burden to the patients, the occurrence of adverse events as abdominal pain and fever, eventually diagnosed as PECS, is associated with the costs of the inpatient treatment, medications and medical imaging. In addition, the steady increase of therapeutic procedures due to the implementation of colorectal cancer screening and colonoscopy surveillance will lead to a rising number of such adverse events. For these reasons, it makes sense to evaluate the possible measures to prevent them. At present, there are no standardized guidelines for the antimicrobial prophylaxis in patients treated with polypectomy, EMR or ESD, so that the antibiotic use often depends on the operators' preference and experience [4].

In our meta-analysis, the use of prophylactic antibiotics in patients with colorectal lesions undergoing endoscopic resection resulted in a statistically significant re- duction of clinical adverse events other than perforation and bleeding. All studies showed a reduction in the rate of such adverse events in patients treated with antibiotics. The pooled analysis showed a reduction of $83 \%$ when antibiotics are administered. The collected data show an advantage in the use of antibiotics to prevent adverse events after the endoscopic resection of colorectal polyps.

On the other hand, in 2014, the World Health Organization reported on the escalating global incidence of multidrug resistance caused by antibiotic overuse, which has become a significant threat to public health worldwide. Use of antibiotics is a risk factor for Clostridium difficile infection. In addition, antibiotic treatment can cause nausea, allergic reactions, and digestive complaints [21]. Another aspect to consider is the increase of costs associated with a standard antibiotic prophylaxis. Consequently, a correct selection of patients who can actually profit from antibiotic prophylaxis is needed. In their multicenter, retrospective study, Cha et al. [2] reported a rate of PECS of $2.9 \%$ after colonoscopic polypectomies and identified hypertension, large size $(>2 \mathrm{~cm})$ and nonpolypoid configuration of the lesion as independent risk factors related to the development of PECS. In addition, this complication seems to occur more often after endoscopic resection of lesions of the right sided colon and in female patients [4]. Thus, it could be reasonable to use an antibiotic prophylaxis in such risk situations, even strong evidences still lack.

Nevertheless, this study has several limitations. First, we only found tree prospective, randomized trial and a retrospective case-control study about this issue. A larger number of works would be ideal to allow an adequate interpretation of the statistical analysis and to provide a more reliable interpretation of the funnel plot. Regarding the sample size, the study of Muro et al. [17] contributed wit $57 \%$ of patients in the treatment group and $35 \%$ of patients in the control group. An analysis omitting this study was performed, and a RR of 0.161 with the random model was achieved (95\% CI 0.0843-0.307, $p<0.001$ ). Moreover, the test for heterogeneity I 2 showed a very low probability $(0 \%)$ of one study-effect with a $p$ value of 0.6173 . These results support the fact that a bias due to a large sample size from this study on our final results was inconsistent. In addition, inclusion criteria differed among studies, being sizes and morphological characteristics not comparable. Nevertheless, no statistically significant differences were noted between antibiotic and control groups in terms of size and polyps morphology. Investigating the role of the antibiotic therapy, it should be said, that even though inclusion criteria are not com- 
parable, the effect of the antibiotic therapy was sought between comparable groups and, therefore, the expected bias could be considered of low relevance. Another limitation is the considerable heterogeneity in definitions of adverse events and antibiotics administration. In fact, Lee et al. [14] considered the electrocoagulation syndrome, Zhang et al. [15] and Shi et al. [16] reported about abdominal pain, fever, leukocytosis, and C-reactive protein and Muro et al. [17] reported the incidence of postoperative infections requiring antibiotic therapy. These differences could represent a bias, so that, the performed quality analysis of the included studies, due to lack of blinding and directness, led to a "low" overall quality of evidence of this meta-analysis according to the GRADE approach [10]. Concerning the risk of publication bias, our metaanalysis adhered to Preferred Reporting Items for Systematic Reviews and Meta-Analyses guidelines, the gray literature was searched in order to find unpublished material but no relevant studies were found. Moreover, the funnel plot did not show evident asymmetries and the Harbord test was not significant $(p=0.274)$. Due to these findings, the risk of publication bias was considered low, even though a degree of uncertainty could remain.

Another issue is the higher incidence of postoperative events reported in the considered studies as compared to larger series [3]. This might be due to differences in definitions of an adverse event. Nevertheless, regardless of the absolute number of postoperative PECS, in all selected studies, we found a statistically significant difference between groups. In addition, we tried to perform a subset analysis of specific patients' subgroups, in order to find particular risk factors of PECS as the abovementioned resection technique, gender, lesion dimension, and location. Unfortunately, no sufficient available data for this purpose was to achieve despite our efforts.

Finally, we should also keep in mind the results of a further study. Min et al. [18] evaluated prospectively the frequency of bacteremia associated with an EMR and ESD for colon lesions in 40 patients (37 EMR and 7 ESD). Blood cultures at baseline and $5 \mathrm{~min}$ after the endoscopic resection were all negative. Only one blood culture at 30 min after an EMR was positive (coagulase-negative Staphylococcus). None of the 40 patients suffered from complications. Despite not having considered this study in our meta-analysis this study due to the lack of the treatment arm, we found fair to mention it, as this work seems to contradict the results of our meta-analysis.

Further studies are needed to confirm our results and to assess subgroups of patients that could actually benefit from an antibiotic prophylaxis.

Antibiotic Prophylaxis in Polyp

Resections

\section{Conclusions}

In our meta-analysis, the antibiotic prophylaxis showed a positive effect in reducing the incidence of postoperative adverse events other than perforation and bleeding in patients treated with EMR and ESD for colorectal lesions. Despite the low-level of evidence of this meta-analysis, the antibiotic prophylaxis should be taken into account. Further multicenter, large-sample, randomized controlled studies are needed to confirm our results and to evaluate whether specific subgroups of patients could actually benefit from an antibiotic prophylaxis.

\section{Statement of Ethics}

Patients' informed consent and Ethical Committee approval were not needed. This original report has not been previously published or submitted elsewhere for publication and will not be sent to another journal until a decision concerning publication is made.

\section{Disclosure Statement}

All authors have no conflicts of interest or financial ties to disclose.

\section{Funding Sources}

No funding were received for this article.

\section{Author Contributions}

M.D.G., A.F., A.F.T.V., G.L.R., and A.S.: protocol/project development. F.M., A.S., M.C., A.F.T.V., G.L.R., and F.G.: data acquisition and interpretation of data. M.C. and F.M.: statistics analysis of data. D.L.R., F.M., A.F.T.V., and F.G.: manuscript drafting. D.L.R., F.M., A.F.T.V., M.D.G., A.S., and F.G.: manuscript Revision and accountable for all aspects of the work.

References

Dig Dis 2020;38:15-22

1 Reumkens A, Rondagh EJ, Bakker CM, Winkens B, Masclee AA, Sanduleanu S. PostColonoscopy Complications: A Systematic Review, Time Trends, and Meta-Analysis of Population-Based Studies. Am J Gastroenterol. 2016 Aug;111(8):1092-101.

2 Cha JM, Lim KS, Lee SH, Joo YE, Hong SP, Kim TI, et al. Clinical outcomes and risk factors of post-polypectomy coagulation syndrome: a multicenter, retrospective, casecontrol study. Endoscopy. 2013;45(3):202-7. 
3 Hirasawa K, Sato C, Makazu M, Kaneko H, Kobayashi R, Kokawa A, et al. Coagulation syndrome: delayed perforation after colorectal endoscopic treatments. World J Gastrointest Endosc. 2015 Sep;7(12):1055-61.

4 Yamashina T, Takeuchi Y, Uedo N, Hamada K, Aoi K, Yamasaki Y, et al. Features of electrocoagulation syndrome after endoscopic submucosal dissection for colorectal neoplasm. J Gastroenterol Hepatol. 2016 Mar; 31(3):615-20.

5 Arimoto J, Higurashi T, Kato S, Fuyuki A, Ohkubo H, Nonaka T, et al. Risk factors for post-colorectal endoscopic submucosal dissection (ESD) coagulation syndrome: a multicenter, prospective, observational study. Endosc Int Open. 2018 Mar;6(3):E342-9.

6 Jung D, Youn YH, Jahng J, Kim JH, Park H. Risk of electrocoagulation syndrome after endoscopic submucosal dissection in the colon and rectum. Endoscopy. 2013 Sep;45(9):714-7.

7 Tang X, Gong W, Jiang B. Antibiotic prophylaxis for GI endoscopy. Gastrointest Endosc. 2015 Jun;81(6):1503-4.

8 Moher D, Liberati A, Tetzlaff J, Altman DG; PRISMA Group. Preferred reporting items for systematic reviews and meta-analyses: the PRISMA statement. J Clin Epidemiol. 2009 Oct;62(10):1006-12.
9 Study Quality Assessment of Controlled Intervention Studies NHLBI. National Heart, Lung, and Blood Institute; National Institutes of Health; U.S. Department of Health and Human Services. https://www.nhlbi.nih.gov/ health-topics/study-quality-assessmenttools.

10 Balshem $\mathrm{H}$, Helfand $\mathrm{M}$, Schünemann $\mathrm{HJ}$, Oxman AD, Kunz R, Brozek J, et al. GRADE guidelines: 3 . Rating the quality of evidence. J Clin Epidemiol. 2011 Apr;64(4):401-6.

11 DerSimonian R, Laird N. Meta-analysis in clinical trials. Control Clin Trials. 1986 Sep; 7(3):177-88.

12 Higgins JP, Thompson SG. Quantifying heterogeneity in a meta-analysis. Stat Med. 2002 Jun;21(11):1539-58.

13 Harbord RM, Harris RJ, Sterne JA. Updated tests for small-study effects in meta-analyses. Stata J. 2009;9(2):197-210.

14 Lee SP, Sung IK, Kim JH, Lee SY, Park HS, Shim CS, et al. A randomized controlled trial of prophylactic antibiotics in the prevention of electrocoagulation syndrome after colorectal endoscopic submucosal dissection. Gastrointest Endosc. 2017 Aug;86(2):349-357.e2.

15 Zhang QS, Han B, Xu JH, Gao P, Shen YC. Antimicrobial prophylaxis in patients with colorectal lesions undergoing endoscopic re- section. World J Gastroenterol. 2015 Apr; 21(15):4715-21.

16 Shi Z, Qiu H, Liu H, Yu H. Should antibiotics be administered after endoscopic mucosalresection in patients with colon polyps? Turk J Med Sci. 2016 Nov;46(5):1486-90.

17 Muro T, Higuchi N, Imamura M, Nakagawa $\mathrm{H}$, Honda M, Nakao K, et al. Post-operative infection of endoscopic submucosal dissection of early colorectal neoplasms: a case-controlled study using a Japanese database. J Clin Pharm Ther. 2015 Oct;40(5):573-7.

18 Min BH, Chang DK, Kim DU, Kim YH, Rhee PL, Kim JJ, et al. Low frequency of bacteremia after an endoscopic resection for large colorectal tumors in spite of extensive submucosal exposure. Gastrointest Endosc. 2008 Jul; 68(1):105-10.

19 Wallace MB. New Strategies to Improve Polypectomy During Colonoscopy. Gastroenterol Hepatol (N Y). 2017 Oct;13(10 Suppl 3):112.

20 Waye JD. The postpolypectomy coagulation syndrome. Gastrointest Endosc. 1981;27(3): 184

21 Llor C, Bjerrum L. Antimicrobial resistance: risk associated with antibiotic overuse and initiatives to reduce the problem. Ther Adv Drug Saf. 2014 Dec;5(6):229-41. 